\title{
Programmable one-pot multistep organic synthesis using DNA junctions
}

\author{
Mireya L. McKee ${ }^{\dagger}$, Phillip J. Milnes ${ }^{\ddagger}$, Jonathan Bath ${ }^{\dagger}$, Eugen Stulz ${ }^{\S}$, Rachel K. O’Reilly ${ }^{\ddagger}$, and Andrew \\ J. Turberfield ${ }^{\dagger, *}$
}

${ }^{\dagger}$ Department of Physics, University of Oxford, Clarendon Laboratory, Parks Road, Oxford, OX1 3PU, UK

${ }^{\ddagger}$ Department of Chemistry, University of Warwick, Gibbet Hill Road, Coventry, CV4 7AL, UK

${ }^{\S}$ School of Chemistry, University of Southampton, Highfield, Southampton, SO17 1BJ, UK

Supporting Information Placeholder

\begin{abstract}
A system for multi-step DNA-templated synthesis is controlled by the sequential formation of DNA junctions. Reactants are attached to DNA adapters which are brought together by hybridization to DNA template strands. This process can be repeated to allow sequence-controlled oligomer synthesis while maintaining a constant reaction environment, independent of oligomer length, at each reaction step. Synthesis can take place in a single pot containing all required reactive monomers. Different oligomers can be synthesized in parallel in the same vessel, and the products of parallel synthesis can be ligated, reducing the number of reaction steps required to produce an oligomer of a given length.
\end{abstract}

DNA-templated chemistry offers a simple and efficient means to control the synthesis of oligomers. DNA hybridization can trigger the coupling of monomers attached to oligonucleotide adapters by increasing their effective molarity and thus reaction rate; cross-reactions between other monomers, also present in solution but attached to adapters with unrelated nucleotide sequences, are minimized. ${ }^{1-9}$ Systems in which each type of monomer is identified by a unique DNA adapter allow multi-step synthesis of oligomers using linear templates, ${ }^{10-13}$ branched junctions, ${ }^{14}$ DNA walkers, ${ }^{15}$ or strand-displacement mechanisms. ${ }^{16,17}$ It is desirable to be able to program the simultaneous synthesis of different oligomers in a single reaction vessel, allowing for the parallel synthesis of diverse products. ${ }^{1,3,11,14}$ One of the challenges in multi-step systems is the maintenance of constant reactivity after each reaction step to maximize the potential product length. We have described a system for sequence-controlled oligomer synthesis, based on DNA strand displacement, that does not restrict the number of coupling reactions attempted. ${ }^{16}$ Here, we explore a new DNAtemplated mechanism that also permits all reactive monomers to be present simultaneously in one pot. Synthesis is controlled by the sequential addition of instruction strands. This confers significant advantages over systems which require sequential addition of reactants. The method also permits programmable parallel synthesis of multiple products.

The system consists of oligonucleotide adapters modified with reactive monomers at either the $5^{\prime}$ or $3^{\prime}$ end. Each type of DNA adapter has a unique address domain that identifies the monomer that it carries, and one of two short, complementary, universal domains adjacent to the attached monomer. Reactions are initiated and programmed by addition of template strands that carry two address domains that are complementary to those in two specific adapters. Hybridization of the selected adapters to the template holds reactive monomers in close proximity; subsequent hybridization of their universal domains forms a transient 3-way junction in which the monomers are positioned in an end-of-helix configuration which favors the coupling reaction ${ }^{18,19}$ (Figure 1a). Unpaired thymines, two between the address and universal domains of the adapters and one between the address domains of the template, are included in order to increase the stability of the junction. ${ }^{20,21}$ The DNA template has an overhanging single-stranded toehold ${ }^{22}$ that allows it to be displaced by addition of a complementary removal strand, generating a double-stranded waste product and releasing the reaction product. Coupling of further subunits is programmed by the addition of further template and remover strands, alternately placing the next monomer in the sequence in the correct position to react with the growing chain then removing the spent DNA template and adapter. The final product can be separated from the reaction mixture using a biotinylated strand that is complementary to the final adapter: no intermediate purification steps are necessary.

The monomers have aldehyde and ylide functionalities that allow them to be coupled by the Wittig reaction: ${ }^{12,16}$ nucleophilic attack of the phosphonium ylide of one monomer on the aldehyde of another detaches the growing chain from its DNA adapter and couples it to the incoming monomer through an olefin linkage. As in the ribosome, this pattern of coordinated cleavage and chain transfer to the incoming unit ensures that each reaction occurs in the same environment - in this case, between the aldehyde of the incoming monomer and the ylide of the previously added unit at the base of the growing chain. Figure 1 shows the initial steps of chain growth, starting with a monofunctional (ylide) initiator and proceeding by addition of bifunctional monomers in a sequence determined by the addition of template and removal strands. The reaction is 


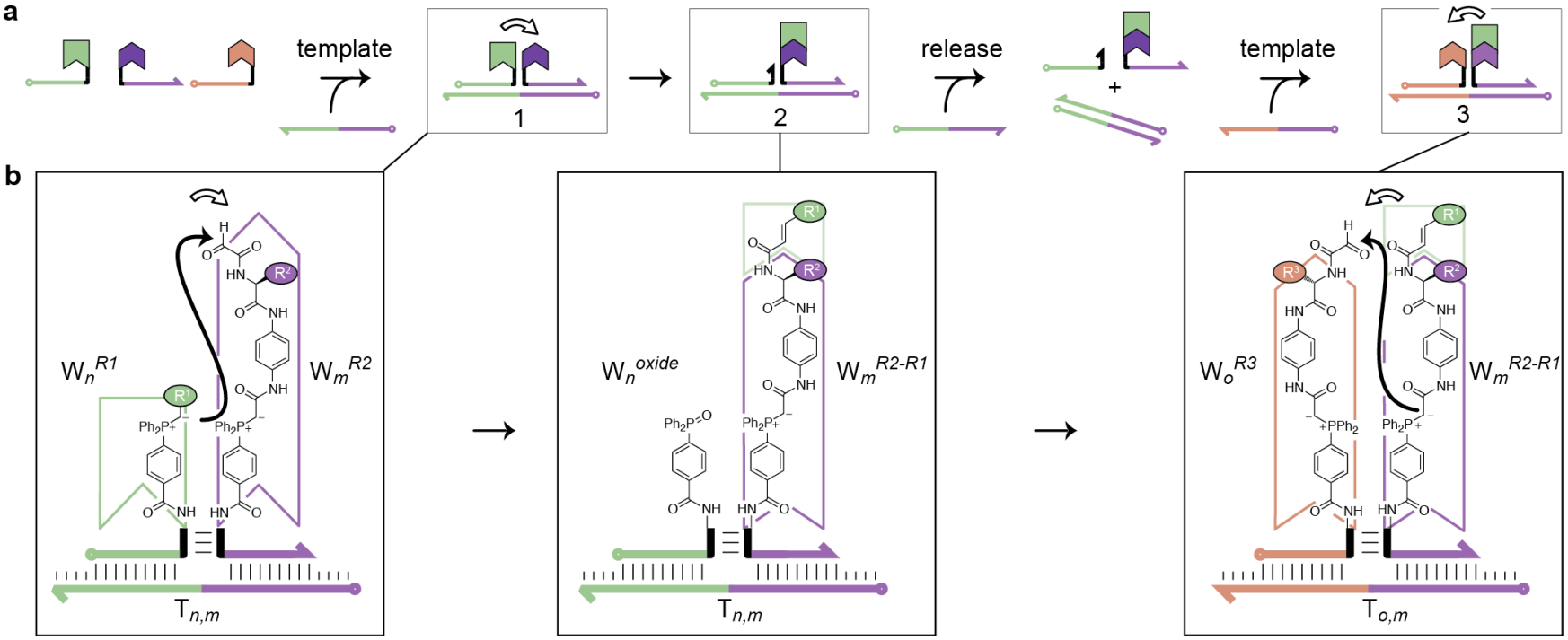

Figure 1. Mechanism for sequence-controlled oligomer synthesis. Reactive monomers are attached to DNA adapters whose nucleotide sequences encode the identities of the monomers. Monomers with both aldehyde and ylide groups are represented by chevrons: the symbol capped at the top represents a monofunctional (ylide) initiator. a Addition of a template strand binds together a specific pair of monomers, enabling the coupling reaction (indicated by the white arrow). Sequential addition of template and removal strands allows sequencecontrolled oligomer growth. b. Chemistry of labeled intermediates, with arrows indicating nucleophilic attack of the incoming ylide on the aldehyde of the previously added monomer that results in cleavage and transfer of the growing chain.

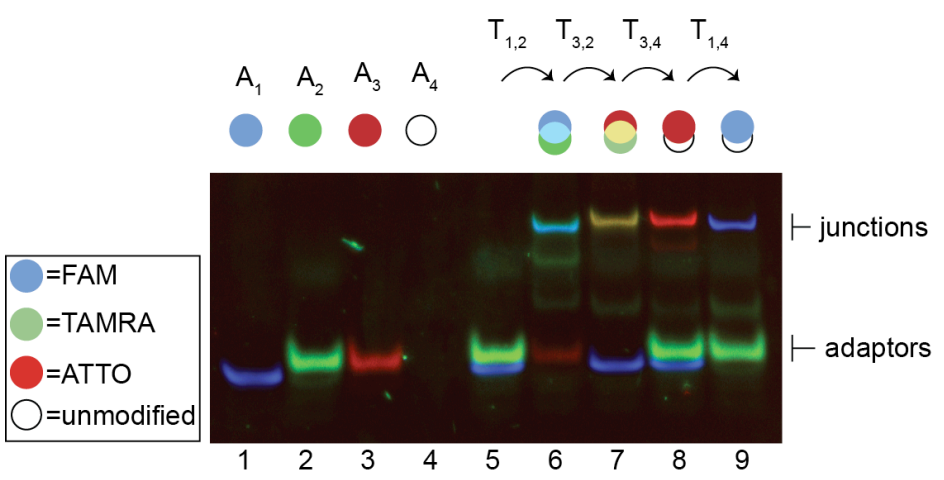

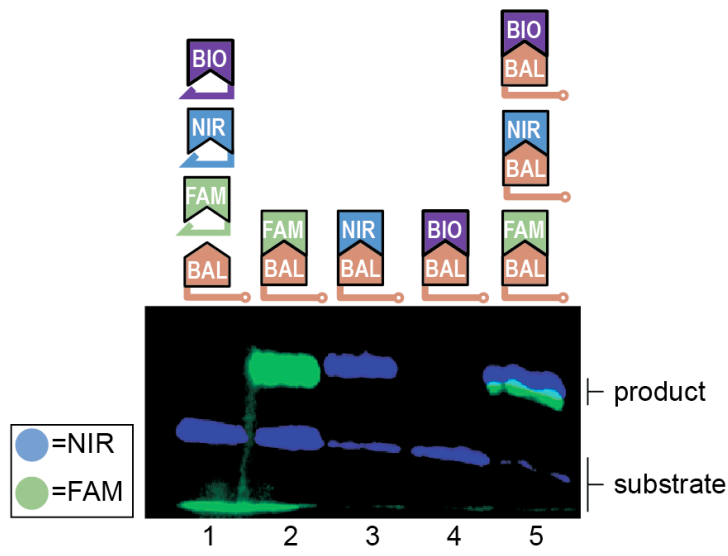

Figure 2. 'Template and release' mechanism allows control of sequential pairwise interactions. a Demonstration of template and release mechanism using fluorescently labelled adaptors, analyzed by PAGE. Adapters $\mathrm{A}_{1-3}$ were modified with fluorescent groups FAM, TAMRA and ATTO647; $\mathrm{A}_{4}$ was unmodified. The image of the $20 \%$ non-denaturing gel is an overlay of three channels: blue (FAM) $488 / 530 \mathrm{~nm}$, green (TAMRA) 532/605 nm and red (ATTO) 635/695nm. Individual channels are shown in Supporting Information. b Specific single-step reactions, controlled out by addition of template strands to a mixture of adapters. The reaction mix contains the start adapter $\mathrm{W}_{4}{ }^{\mathrm{BAL}}$ and three stop adapters $\mathrm{W}_{1}{ }^{\mathrm{FAM}}, \mathrm{W}_{9}{ }^{\mathrm{NIR}}, \mathrm{W}_{3}{ }^{\mathrm{BIO}}$ (lane 1). Addition of templates $\mathrm{T}_{1,4} \mathrm{~T}_{9,4}$ and $\mathrm{T}_{3,4}$ (lanes 2-4) results in the formation of dimer products BAL-FAM, BAL-NIR, BAL-BIO respectively. Simultaneous addition of all three templates results in parallel synthesis of all three dimers (lane 5). The image of the $20 \%$ denaturing gel is an overlay of two channels: green (FAM) $488 / 530 \mathrm{~nm}$ and blue (NIR) 635/695 $\mathrm{nm}$.

terminated when a monomer containing only an aldehyde is incorporated (not shown). Each monomer incorporates a characteristic functional unit which here consists of an amino acid residue or a fluorophore. The structures of the monomers and their synthesis are described in Supporting Information. We use symbol $\mathrm{A}_{n}$ to denote DNA adapter sequence $n$ and $\mathrm{W}_{n}{ }^{R}$ to denote the corresponding adapter carrying a reactive (Wittig) monomer incorporating functional group $R$.

All reactive monomers can be present in the same solution: they will not react at a significant rate until they are linked by hybridization to the correct template because the universal sequence is too short to promote stable binding between DNA adapters in the absence of a template. The technique can therefore be applied to parallel multistep reactions and has the potential to create complex oligomer libraries in one pot.

The use of template and removal strands to control association between monomers was tested using dummy adapter strands $A_{1}-A_{4}$ coupled to fluorophores (Figure 2a). Nondenaturing polyacrylamide gel electrophoresis (PAGE) shows that there is no interaction between the adapters in solution in the absence of template (Figure 2a, lane 5). Addition of template $\mathrm{T}_{1,2}$ generates a band containing both FAM and TAMRA 
corresponding to the 3 -strand complex $\mathrm{A}_{1}{ }^{\mathrm{FAM}} \cdot \mathrm{A}_{2}{ }^{\mathrm{TAMRA}} \cdot \mathrm{T}_{1,2}$ (Figure 2a, lane 6). Subsequent addition of the removal instruction $\mathrm{R}_{1,2}$ (complementary to $\mathrm{T}_{1,2}$ ) and a second template instruction $\left(\mathrm{T}_{3,2}\right)$ releases $\mathrm{A}_{1}^{\mathrm{FAM}}$ and generates a new band corresponding to $\mathrm{A}_{2}{ }^{\text {TAMRA }} \cdot \mathrm{A}_{3}{ }^{\text {ATTO }} \cdot \mathrm{T}_{3,2}$ (Figure $2 \mathrm{a}$, lane 7 ). Similarly, addition of $\mathrm{R}_{3.2}$ and $\mathrm{T}_{3.4}$ and then $\mathrm{R}_{3.4}$ and $\mathrm{T}_{1.4}$ generates

a

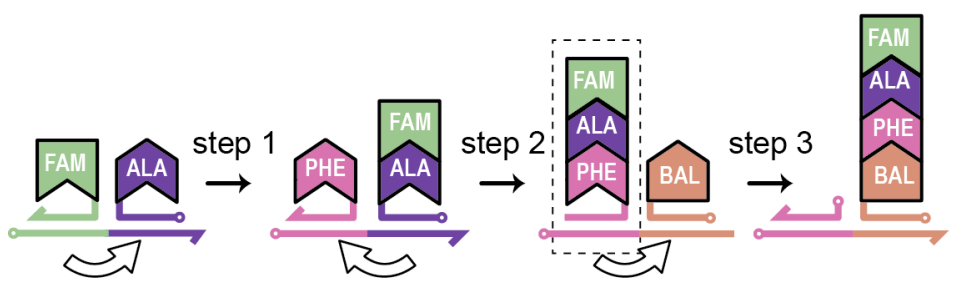

b
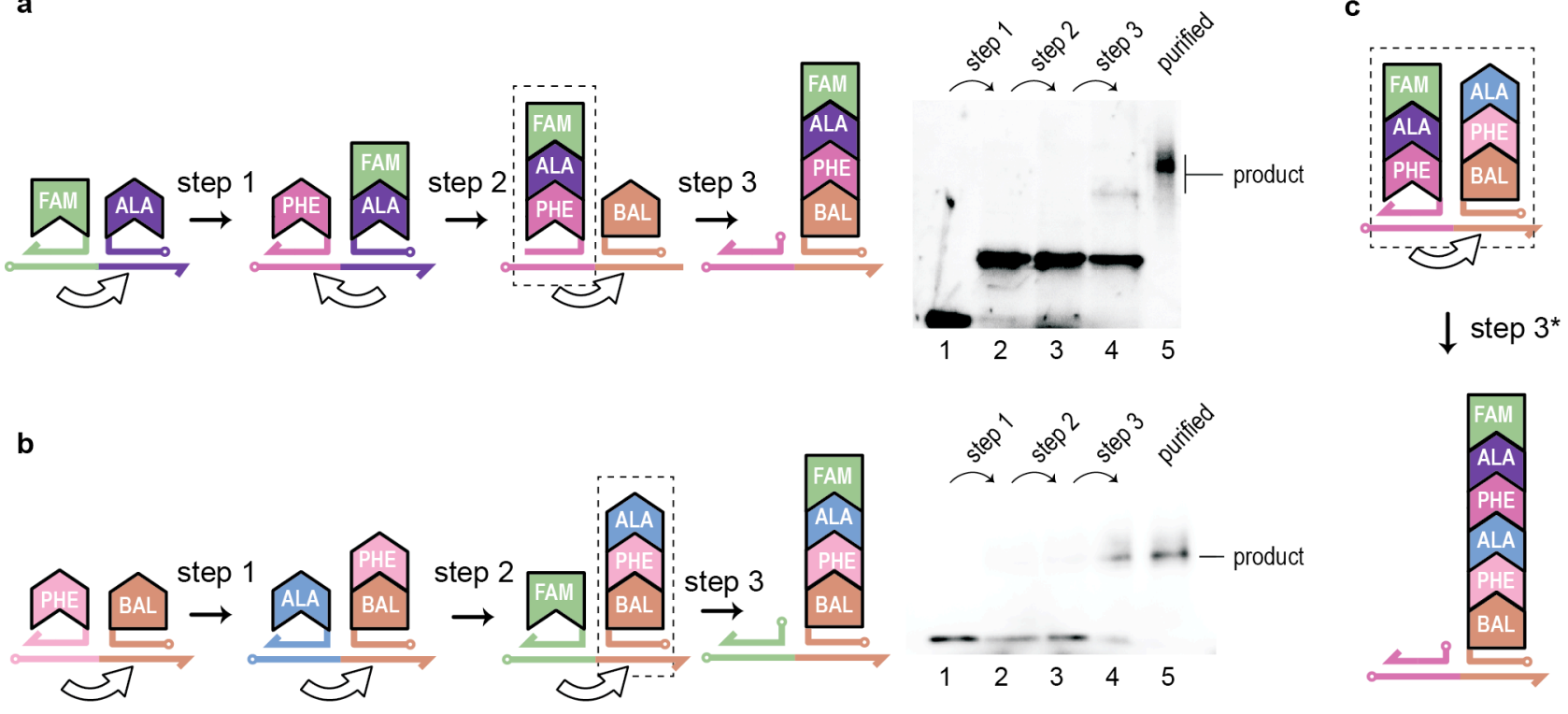

d

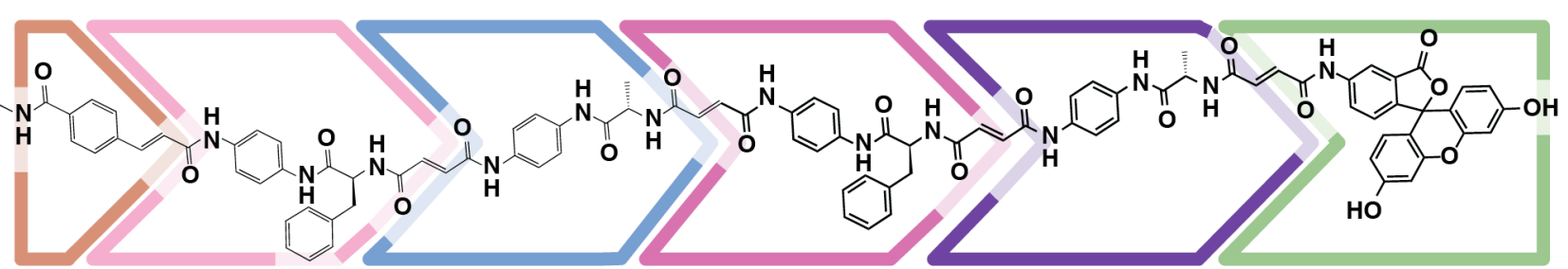

Figure 3. Two mechanisms for the synthesis of oligomers. a The alternating-strand mechanism is initiated with a monofunctional ylide monomer (FAM). At each step, the growing chain is transferred to the incoming monomer. The reaction is terminated by transfer to a monofunctional aldehyde monomer (BAL). Adapters $\mathrm{W}_{1}{ }^{\mathrm{FAM}}, \mathrm{W}_{2}{ }^{\mathrm{ALA}}, \mathrm{W}_{3}{ }^{\mathrm{PHE}}$ and $\mathrm{W}_{4}{ }^{\mathrm{BAL}}$ with template/removal pairs $\left(\mathrm{T}_{1,2} / \mathrm{R}_{1 / 2}, \mathrm{~T}_{3,2} / \mathrm{R}_{3,2}\right.$ and $\mathrm{T}_{3,4} / \mathrm{R}_{3,4}$ ) were used. b The same-strand mechanism is initiated with an aldehyde monomer: the growing chain remains on the same adapter throughout the synthesis and the chain is terminated by addition of an ylide monomer (FAM). Adapters $\mathrm{W}_{4}{ }^{\mathrm{BAL}}, \mathrm{W}_{5}{ }^{\mathrm{ALA}}, \mathrm{W}_{7}{ }^{\mathrm{PHE}}$ and $\mathrm{W}_{1}{ }^{\mathrm{FAM}}$ with template/removal pairs $\left(T_{7,4} / R_{7 / 4}, T_{5,4} / R_{5,4}\right.$ and $\left.T_{1,4} / R_{1,4}\right)$ were used. In both cases , the mixture of adapters (lane 1), intermediates of stepwise synthesis (lanes 2-4) and purified products (lane 5) were analyzed on a $20 \%$ denaturing gel scanned at $488 / 530 \mathrm{~nm}$ em/ex to reveal FAM fluorescence. c By coupling products generated by the two mechanisms simultaneously, in the same reaction vessel, the number of steps required to make a given product can be halved. Here, a 6-mer, d, was made in three operations (Supporting Information): the 3mers boxed with dotted lines in $\mathbf{a}$ and $\mathbf{b}$ were produced in parallel and then ligated using template $\mathrm{T}_{3,4}$.

This system allows more than one reaction sequence to be programmed simultaneously in a single reaction vessel. Control of parallel reactions is possible because no significant reactions occur in the absence of template strands. Initial experiments were performed using the coupling of monofunctional monomers to form olefin dimers. Three different ylide adapters and one aldehyde adapter were used (Figure $2 b)$ : two of the ylides contained fluorescent tags $\mathrm{W}_{1}{ }^{\mathrm{FAM}}$ and $\mathrm{W}_{9}{ }^{\mathrm{NIR}}$ and the third, $\mathrm{W}_{3}{ }^{\mathrm{BIO}}$, contained a biotin group. Transfer of these monomers to the longer aldehyde adapter was monitored using denaturing PAGE. Addition of individual template strands $\mathrm{T}_{1,4}, \mathrm{~T}_{9,4}$ or $\mathrm{T}_{3,4}$ produced the intended products (Figure 2b, lanes 2-4) whereas simultaneous addition all three template strands together with three equivalents of $\mathrm{W}_{4}{ }^{\mathrm{BAL}}$ resulted in formation of all three olefin dimers (Figure $2 \mathrm{~b}$, lane 5; generation of the non-fluorescent BAL-BIO dimer was confirmed by MS).
Multistep reactions can be programmed with either a monofunctional ylide or an aldehyde monomer as the chain initiator. When an ylide is used as the initiator (Figure 1b) the growing chain is transferred between successive adapters, alternating between $3^{\prime}$ and $5^{\prime}$ ends (alternating-strand mechanism). With an aldehyde initiator, the oligomer product remains covalently attached to the initial adapter throughout the series of reactions (same-strand mechanism, Figure S5). The same-strand mechanism does not preserve a constant reaction environment (the reactive aldehyde is at the distal end of the growing chain) but it does offer the advantage that the adapter bearing the oligomer can always be unambiguously identified, allowing the use of a single adapter sequence for a monomer that is to be incorporated at more than one site.

Both mechanisms were tested by programming the synthesis of 4-mers (Figure 3). To facilitate analysis by PAGE, adapters with different sizes from 20 to 32 nucleotides were 
used (see Supporting Information for nucleotide sequences). In both syntheses the adapter to which the final product is attached, $\mathrm{W}_{4}{ }^{\mathrm{BAL}}$, is longer than other adapters. The monofunctional ylide adapter $\mathrm{W}_{1}{ }^{\mathrm{FAM}}$ used to initiate the alternating-strand synthesis and to terminate the same-strand synthesis contains the fluorescent group FAM, allowing reaction progress to be monitored. Transfer between adapters of the fluorescent tag was observed after the first step using the alternating-strand mechanism (Figure 3a, lane 2); using the same-strand mechanism, there was no significant change in mobility of the fluorescent band until the last step (Figure $3 \mathrm{~b}$, lane 4 ), as expected. The identity of each product was confirmed by mass spectrometry (see Supporting Information for MS and yield estimates). Incomplete products but no out-of-sequence monomer additions were observed when the products of each step in the same-strand mechanism were analyzed. The main cause of the formation of truncated products is oxidation of the ylide. ${ }^{12}$ Parallel multistep chemistry was also tested by synthesizing two different 4-mers in a single reaction vessel (Supporting Information). These results are consistent with the successful operation of both variants of the sequence-controlled synthesis mechanism.

The number of reaction steps required to make an oligomer of a given length can be reduced by synthesizing sections in parallel. This tactic could greatly increase the complexity of product libraries and simplify synthetic schemes. As proof of principle, a 6-mer was synthesized in 3 reaction steps. Two trimers were synthesized simultaneously in a single vessel, one using the alternating-strand and the other the same-strand mechanism. The two intermediate products were then brought together by a third template strand $\left(\mathrm{T}_{3,4}\right)$ triggering coupling to form the olefin 6-mer (Figure 3c). The resulting products were purified using streptavidin beads and analyzed by PAGE and mass spectrometry. The desired 6mer, with 5-mer, 4-mer and dimer truncated products, was observed (Supporting Information).

We have demonstrated a method for one-pot, sequencecontrolled oligomer synthesis using a DNA-templated reaction mechanism in which all adapters are present but react only when a template instruction strand is introduced. This system has several new and useful characteristics. It allows parallel multistep synthesis in a single reaction vessel. We have demonstrated multistep synthesis using two distinct mechanisms: in the first, the growing chain is transferred between adapters at each step (as in the ribosome); in the second, the growing chain is assembled on a single adapter. Combining the two mechanism halves the number of operations required to make a product of a given length, potentially allowing access to more complex product libraries. The alternating-strand mechanism maintains a constant reaction environment and therefore constant reactivity, independent of oligomer length, at each reaction step.

\section{ASSOCIATED CONTENT}

Supporting Information. Experimental methods and supporting data. This material is available free of charge via the Internet at http://pubs.acs.org

\section{AUTHOR INFORMATION}

Corresponding Author

* e-mail: a.turberfield@physics.ox.ac.uk
M.L.M., P.J.M, and J.B. carried out the experimental work. All the authors contributed to the design of the experiments, data analysis and writing the manuscript.

\section{ACKNOWLEDGMENT}

This work was supported by EPSRC IDEAS Factory grants EP/F056605/1, EP/008597/1 and linked grants

\section{ABBREVIATIONS}

FAM, fluorescein; TAMRA, tetramethylrhodamine; BIO, biotin; BAL, benzaldehyde.

\section{REFERENCES}

(1) Gartner, Z. J.; Liu, D. R. J. Am. Chem. Soc. 2001, 123 , 6961-6963.

(2) Gartner, Z, J.; Kanan, M. W.; Liu, D. R. Angew. Chem. Int. Ed., 2002, 41, 1796-1800.

(3) Calderone, C. T.; Puckett, J. W.; Gartner, Z. J.; Liu, D. R. Angew. Chem. Int. Ed. 2002, 41, 4104-4108.

(4) Li, X.; Liu, D. R. Angew. Chem. 2004, 43, 4848-4870

(5) Chhabra, R.; Sharma, J.; Liu, Y.; Yan, H. Nano Lett., 2006, 6, 978-983.

(6) Grossmann, T.N.; Seitz, O. J. Am. Chem. Soc. 2006, 128, 15596-15597.

(7) Jacobsen, M.F.; Cló, E.; Mokhir, A.; Gothelf, K.V. ChemMedChem 2007, 2, 793-799.

(8) Scheuermann, J.; Dumelin C.E.; Melkko, S.; Neri, D. J. Biotech. 2006, 126, 568-581.

(9) Ravnsbæk, J.B.; Jacobsen, M.F.; Rosen, C. B.; Voigt, N.B.; Gothelf K.V. Angew. Chem. Int. Ed. 2011, 50, 10851-10854.

(10) Gartner, Z. J.; Kanan, M. W.; Liu, D. R. J. Am. Chem. Soc. 2002, 124, 10304-10306.

(11) Gartner, Z. J.; Tse, B. N.; Grubina, R.; Doyon, J. B.; Snyder, T. M.; Liu, D. R. Science 2004, 305, 1601-1605.

(12) Snyder, T. M.; Liu, D. R. Angew. Chem. Int. Ed. 2005, 44, 7379-7382.

(13) Li, X. Y.; Gartner, Z. J.; Tse, B. N.; Liu, D. R. J. Am Chem. Soc. 2004, 126, 5090-5092.

(14) Hansen, M. H.; Blakskjaer, P.; Petersen, L. K.; Hansen, T. H.; Hojfeldt, J. W.; Gothelf, K. V.; Hansen, N. J. V. J. Am. Chem. Soc. 2009, 131, 1322-1327.

(15) He, Y.; Liu D. R. Nat. Nanotechnol., 2010, 5, 778-782.

(16) McKee, M.; Milnes, P.; Bath, J.; Stulz, E.; Turberfield, A. J.; O'Reilly, R. K. Angew. Chem. Int. Ed. 2010, 49, 7948-7951.

(17) He, Y.; Liu, D. R. J. Am. Chem. Soc. 2011, 133, 9972 9975.

(18) McKee, M. L.; Evans, A. C.; Gerrard, S. R.; O’Reilly, R. K.; Turberfield, A. J.; Stulz, E. Org. Biomol. Chem. 2011, 9, 16611666.

(19) Gartner, Z. J.; Grubina, R.; Calderone, C. T.; Liu, D. R. Angew. Chem. Int. Ed. 2003, 42, 1370-11375.

(20) Leontis, N. B.; Kwok, W.; Newman, S. Nucleic Acids Res. 1991, 19, 759-766.

(21) Zhong, M.; Michael, S.; Rashes, M. S.; Leontis, N. B.; Kallenbach, N. R. Biochemistry 1994, 33, 3660-3667.

(22) Yurke, B.; Turberfield, A. J.; Mills, A. P., Jr.; Simmel, F. C.; Neumann, J. L. Nature 2000, 406, 605-608.

\section{Author Contributions}


Insert Table of Contents artwork here

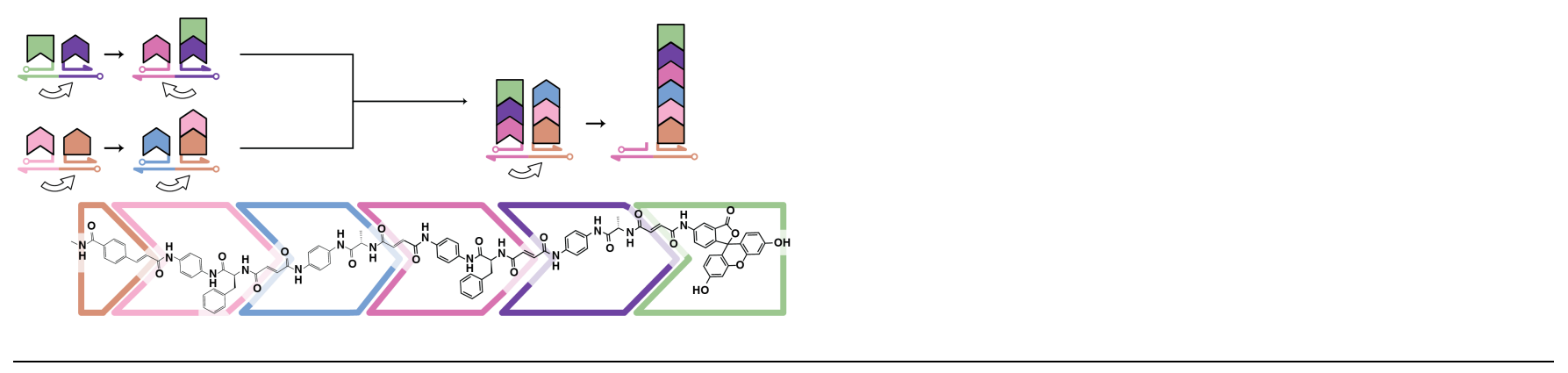

5 